\title{
Research of Mechanochemical Processes of Activation of Building Materials by Air-Impact Method
}

\author{
Igor Kravchenko ${ }^{1}$, Mihail Erofeev ${ }^{1}$, Aleksandr Fedorov $^{2}$, Valeriy Kondrashchenko ${ }^{3}$, Diana Abdumuminova ${ }^{4 *}$, Shukurilo \\ Yuldashev $^{4}$, Jaloliddin Matyokubov $^{5}$, and Kudrat Bobomurodov ${ }^{6}$ \\ ${ }^{1}$ Institute of Mechanical Engineering named after A.A. Blagonravov Russian Academy of Sciences, Moscow, Russia \\ ${ }^{2}$ Military Academy of Material and Technical Support Army General A.V. Khruleva, St. Petersburg, Russia \\ ${ }^{3}$ Russian University of Transport (MIIT), Moscow, Russia \\ ${ }^{4}$ Tashkent Institute of Irrigation and Agricultural Mechanization Engineers, Tashkent, Uzbekistan \\ ${ }^{5}$ Uzbekistan State World Language University Tashkent, Uzbekistan \\ ${ }^{6}$ Termez State University, Termez, Surkhandarya, Uzbekistan
}

\begin{abstract}
To increase the specific surface area of the binder and accelerate the strength gain of fast-hardening concrete mixtures, a model has been created for calculating aeroactivators as part of mobile complexes based on new equipment for cement activation, sand enrichment, local materials, and production waste. The proposed model establishes the dependence of the rate of destruction of the dusty shell of particles on the mechanical impact of the beaters and materials' physical and mechanical characteristics. To improve the operational characteristics of the cement aeroactivator, experimental studies were carried out, the results of which made it possible to determine the rational parameters of the developed installation. Based on the test results, it was found that the use of the cement activation process with additives accelerates the hardening process of concrete and mortar mixtures in the initial period, increases the design strength of concretes and mortars by $20-40 \%$ in comparison with the strength of the control composition at the same cement consumption, and also reduces the consumption cement up to $17 \%$ to obtain concretes and mortars equal to the control composition at the design age.
\end{abstract}

\section{Introduction}

In remote, hard-to-reach areas with harsh climatic conditions, the construction of housing and infrastructure facilities is impossible without the use of activated (additional grinding and grinding) local building materials and technological equipment with effective characteristics [1-4].

The development of technological equipment for mobile construction complexes about the local resource base, natural and climatic conditions should be carried out using modern methods of mathematical programming and computer-aided design tools based on multi-criteria analysis [5,6].

Although the methods of activating Portland cement are well studied, confirmed by numerous studies, and supplemented by practical results, in particular, by increasing the fineness of its grinding, they are not widely used due to the lack of applied methods for calculating effective small-sized equipment used in mobile construction complexes. In modern construction, grinding and activation are carried out using various mills and crushers [7-9], which are material and energy-intensive, which makes their use for construction conditions not justified.

The purpose of the research was to substantiate the design parameters of the working bodies (the number of working zones and propeller blades) and to determine the rational operating modes of small-sized activators for cleaning and mechanochemical activation of cement and dry mixtures.

Following the set goal, the following tasks have been identified: a) determination of the degree of activation by comparing the fractional composition of the original and activated cement by the residue on the sieve; b) study of the physical characteristics of quick-hardening concretes prepared based on activated cement; c) carrying out full-scale tests of the developed installation of the activator.

\section{Methods and Materials}

Methods of systemic, statistical, and multivariate analysis and physical, mathematical, and simulation modeling [10, 11] were used as a research tool for solving the set tasks. The research was carried out using the developed cement aero-

${ }^{*}$ Corresponding author: temurlanovnadiana@gmail.com 
activator (Fig. 1), the distinctive advantages of which are: low specific power consumption; reliability and compactness of the design; high performance.

Based on the proposed aerodynamic activator [12], a technological line for the production of a strengthening plasticizing additive UPD to cement mixtures (concrete, mortar, dry mixture) was installed. The effectiveness of this hardening additive has been tested at several operating enterprises for the production of ready-mixed concrete and dry general construction mixture

The degree of cement activation was controlled by changing the magnitude of the energy effects carried out by an electronic device mounted in a certain working area of the activator grinding chamber.

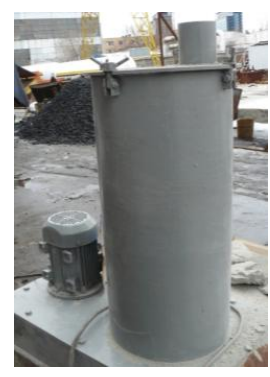

$a$

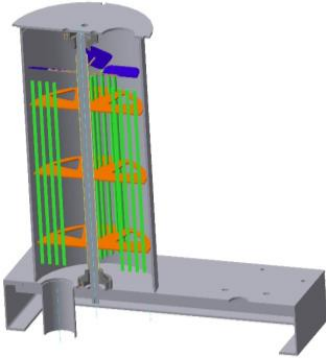

$b$

Fig. 1. A prototype of a cement aeroactivator: $a$ - general view; $b$ - sectional model of the sample

\section{Results and Discussion}

\subsection{Choosing a Rational Number of Work Areas}

When carrying out this experiment, the number of working zones of the activator was taken as a variable parameter, the residence time of the cement in the working zone of the activator, the volume of cement that passed the activation, and the fineness of its grinding was measured.

In the first experiment, the number of working zones of the activator was 2 . In subsequent experiments, the number of working zones was increased by one until the conditions of the experiment were satisfied.

The cement activated in this way was packed in sealed packages and sent to the laboratory for quality control of materials to determine the degree of activation and further research of physical and chemical parameters, as well as quick-hardening, concretes prepared on its basis.

The results of the analysis of experimental studies showed that the cement milled in an activator with two working zones had a coarse-dispersed structure and did not meet the required quality indicators of the fineness of cement grinding - a specific surface area of the order of $4500-6000 \mathrm{~cm}^{2} / \mathrm{g}$.

\subsection{Choosing a Rational Airscrew Design}

The variable value during these experiments was the number of airscrew blades. During the experiments, the residence time of the cement in the working zone of the activator, the volume of the activated cement, and the fineness of its grinding were measured. Initially, a two-blade propeller was used. In subsequent experiments, the number of blades was increased to 4 and 6 pcs respectively.

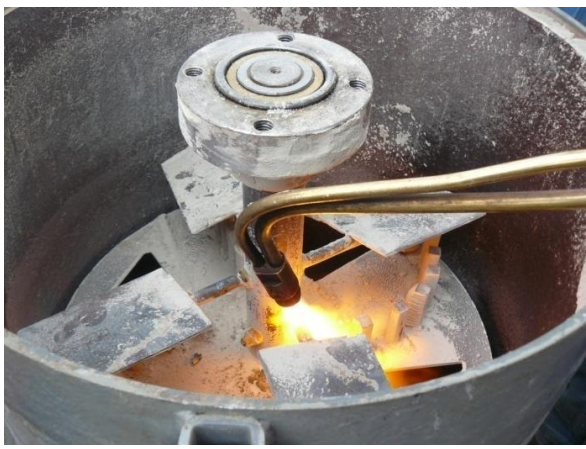

Fig. 2. Varying the number of blades of the propeller design 
For this, starting with 2 blades additional blades were welded on directly at the test site (Fig. 2).

At this stage of the experiment, it can be concluded that the cement milled in an activator with six blades has a coarse structure and does not correspond to the required quality indicators of the fineness of cement grinding - a specific surface area of the order of $4400-5000 \mathrm{~cm}^{2} / \mathrm{g}$.

The analysis of the results showed that the required fineness of grinding was achieved with a rational number of propeller blades equal to four. When the number of blades was less than four, the design pressure of the airflow was not achieved, cement over-grinding due to its relatively long presence, and when the number of blades was more than four, an intensive dusting of cement was observed.

The results of experimental studies on the choice of parameters and operating modes of the activator made it possible to conclude the correctness of the theoretical calculations at the design stage. This served as the basis for the use of an activator of this design for carrying out field tests in the Arctic and further research to improve the operational characteristics of the activator.

\subsection{Research of Activated Cement and Testing of Rapid Hardening Concrete on Its Basis}

Experiments to achieve the performance characteristics of the activated value to select them rationally and to achieve the desired fineness of the activated cement.

Determination of the fineness of cement grinding was carried out using sieve analysis of the fractional composition according to the formula:

$$
T=\left(m_{1} / m\right) \cdot 100 \%
$$

where $m_{1}$ - weight of cement remaining on the sieve, g;

$m$ - weight of the sieved cement sample, $g$.

This research was carried out on prototypes of cement that were activated in the activator during experiments on the choice of a rational number of working zones and the design of the activator's airscrew.

The results of the experiments are shown in the table. 1-3.

For a visual representation, the dependences of the fineness of grinding on the number of working zones of the activator are plotted (Fig. 3).

The studies carried out to determine the fineness of grinding of cement activated in aeroactive mode using different working zones, showed that the best grinding is achieved in an activator, the number of working zones of which is 4 .

Table. 1. The composition of the cement activated in the activator with working zones

\begin{tabular}{|c|c|c|c|c|c|c|c|c|}
\hline \multirow{2}{*}{$\begin{array}{c}\text { Sieve } \\
\text { number }\end{array}$} & \multicolumn{9}{|c|}{ Experimental results } \\
\cline { 2 - 9 } & \multicolumn{2}{|c|}{ № 1 } & \multicolumn{2}{|c|}{ № 2 } & \multicolumn{2}{|c|}{ № 3 } & \multicolumn{2}{c|}{ Mean } \\
\cline { 2 - 9 } & $\mathrm{g}$ & $\%$ & $\mathrm{~g}$ & $\%$ & $\mathrm{~g}$ & $\%$ & $\mathrm{~g}$ & $\%$ \\
\hline 10 & 25.7 & 2.57 & 18.2 & 1.82 & 20.6 & 2.06 & 21.5 & 2.15 \\
\hline 5 & 72.5 & 7.25 & 53.7 & 5.37 & 59.2 & 5.92 & 61.8 & 6.18 \\
\hline 2 & 78 & 7.8 & 69.3 & 6.93 & 65.2 & 6.52 & 70.8 & 7.08 \\
\hline 1 & 105 & 10.5 & 60 & 6.0 & 65.3 & 6.53 & 76.8 & 7.68 \\
\hline 0.5 & 221.7 & 22.17 & 97 & 9.7 & 101 & 10.1 & 139.9 & 13.99 \\
\hline 0.25 & 44.4 & 4.44 & 26.8 & 2.68 & 44.5 & 4.45 & 38.6 & 3.86 \\
\hline 0.1 & 280.3 & 28.03 & 521.2 & 52.12 & 460.1 & 46.01 & 420.5 & 42.05 \\
\hline less 0.1 & 153.1 & 15.31 & 160.3 & 16.03 & 162.1 & 16.21 & 158.5 & 15.85 \\
\hline 0.08 & 19.3 & 1.93 & 20.5 & 2.05 & 22 & 2.2 & 20.6 & 2.06 \\
\hline
\end{tabular}

Table. 2. The composition of the cement activated in an activator with three working zones

\begin{tabular}{|c|c|c|c|c|c|c|c|c|}
\hline \multirow{2}{*}{$\begin{array}{c}\text { Sieve } \\
\text { number }\end{array}$} & \multicolumn{9}{|c|}{ Experimental results } \\
\cline { 2 - 10 } & \multicolumn{2}{|c|}{ № 1 } & \multicolumn{2}{|c|}{ № 2 } & \multicolumn{2}{|c|}{ № 3 } & \multicolumn{2}{c|}{ Mean } \\
\cline { 2 - 10 } & $\mathrm{g}$ & $\%$ & $\mathrm{~g}$ & \% & $\mathrm{g}$ & \% & $\mathrm{g}$ & \% \\
\hline 10 & 5.8 & 0.58 & 10.3 & 1.03 & 5.1 & 0.51 & 7 & 0.7 \\
\hline 5 & 13.7 & 1.37 & 15.1 & 1.51 & 5.7 & 0.57 & 11.5 & 1.15 \\
\hline 2 & 31.2 & 3.12 & 28.7 & 2.87 & 25.1 & 2.51 & 28.3 & 2.83 \\
\hline 1 & 32.3 & 3.23 & 36 & 3.6 & 42.8 & 4.28 & 37 & 3.7 \\
\hline 0.5 & 74.8 & 7.48 & 50.7 & 5.07 & 60.7 & 6.07 & 62 & 6.2 \\
\hline 0.25 & 24.5 & 2.45 & 22.7 & 2.27 & 23.1 & 2.31 & 23.4 & 2.34 \\
\hline 0.1 & 120.3 & 12.03 & 127.1 & 12.71 & 120.5 & 12.05 & 122.6 & 12.26 \\
\hline less 0.1 & 515.1 & 51.51 & 573.1 & 57.31 & 559.4 & 55.94 & 549.2 & 54.92 \\
\hline 0.08 & 182.3 & 18.23 & 136.3 & 13.63 & 147.6 & 14.76 & 155.4 & 15.54 \\
\hline
\end{tabular}


Table. 3. The composition of the cement activated in the activator with four working zones

\begin{tabular}{|c|c|c|c|c|c|c|c|c|c|c|c|c|c|c|}
\hline \multirow{2}{*}{$\begin{array}{c}\text { Sieve } \\
\text { number }\end{array}$} & \multicolumn{10}{c|}{ Experimental results } \\
\cline { 2 - 16 } & \multicolumn{2}{|c|}{ № 1 } & \multicolumn{2}{|c|}{ № 2 } & \multicolumn{2}{c|}{ № 3 } & \multicolumn{2}{|c|}{ № 4 } & \multicolumn{2}{|c|}{ № 5 } & \multicolumn{2}{|c|}{ Mean } \\
\cline { 2 - 15 } & $\mathrm{g}$ & $\%$ & $\mathrm{~g}$ & $\%$ & $\mathrm{~g}$ & $\%$ & $\mathrm{~g}$ & $\%$ & $\mathrm{~g}$ & $\%$ & $\mathrm{~g}$ & \% \\
\hline 10 & - & - & - & - & - & - & - & - & - & - & - & - \\
\hline 5 & - & - & - & - & - & - & - & - & - & - & - & - \\
\hline 2 & 0,3 & 0.03 & 0.2 & 0.02 & 0.2 & 0.02 & - & - & - & - & 0.23 & 0.02 \\
\hline 1 & 2.0 & 0.2 & 3.8 & 0.38 & 2.9 & 0.29 & - & - & - & - & 2.9 & 0.29 \\
\hline 0.5 & 11 & 1.1 & 10.5 & 1.05 & 13.3 & 1.33 & 221.1 & 22.11 & 93.9 & 9.39 & 69.96 & 6.996 \\
\hline 0.25 & 38.6 & 3.86 & 29.1 & 2.91 & 28.5 & 2.85 & 29.9 & 2.99 & 29.3 & 2.93 & 31.72 & 3.17 \\
\hline 0.1 & 24.6 & 2.46 & 76.6 & 7.66 & 49.0 & 4.9 & 200.4 & 20.04 & 137.6 & 13.76 & 97.64 & 9.7 \\
\hline less 0.1 & 194.6 & 19.46 & 160.5 & 16.05 & 173.3 & 17.33 & 94.7 & 9.47 & 143.2 & 14.32 & 153.26 & 15.3 \\
\hline 0.08 & 763.6 & 76.36 & 748.7 & 74.87 & 756.2 & 75.62 & 478.2 & 47.82 & 644.5 & 64.45 & 678.24 & 67.8 \\
\hline
\end{tabular}

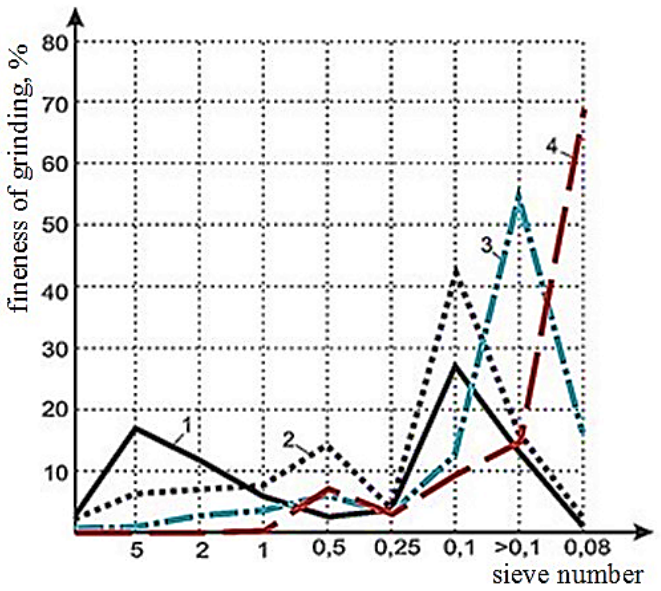

Fig. 3. Dependence of the average fineness cement grinding on the number of working zones of the activator: 1 - non-activated cement (control composition); 2 - two working zones; 3 - three working zones; 4 - four working zones

\subsection{Laboratory Tests of the Developed Activator Installation}

In order to confirm the correctness of analytical studies on the choice of the operating parameters of the aeroactive material, studies are carried out.

In the process of preparing the mixtures, the following parameters were changed: prepared concrete-mortar mixtures; the number of revolutions of the activator rotor shaft by changing the variator of the torque transmission ratio; the volumes of cement passed through the activator and the time of its passage.

Using a laboratory installation (see Fig. 1), concrete mixtures of the following compositions are prepared:

- control composition - based on non-activated cement;

- composition No. 1 - based on activated cement;

- composition No. 2 - based on an activated mixture of cement with the addition of $0.3 \%$ dihydrate gypsum based on the mass of cement;

- composition No. 3 - based on an activated mixture of cement with the addition of 3.0\% dihydrate gypsum based on the mass of cement.

In the process of testing mixtures No. 1, 2, 3, and in the initial period of their hardening, a large heat release was observed in comparison with the control composition. This process is formed by the specific surface area of the binder grains and, as a result, has the highest exotherm in comparison with other components of the cement clinker. Control solutions were molded from the mixtures of each formulation.

The analysis carried out in terms of efficiency indicators showed that, with the help of the equipment used, an aerodynamic activator of increased efficiency due to the use of centrifugal shock forces created by working workers (beats) and cutting edges of the disc, which makes it possible to intensify the process of activation of building

\section{Conclusion}

In the course of experiments to determine the rational parameters of the activator, followed by laboratory studies of activated cement and concretes on their basis, the rational design of the installation to achieve the certain fineness of cement grinding. 
Full-scale tests showed that due to the use of a cement aeroactivator as part of a technological line for the preparation of cement-sand mortars, the mixture laid in the structure gained the required strength faster than usual by $20-30 \%$. This allows you to reduce the overall time of the main structures and structures

\section{References}

1. S.V. Maksimov, E.A. Derbakova, The effectiveness of regional production of dry building mixtures based on local raw materials, Bulletin of the Ulyanovsk State Technical University 2(38), 56-61 (2007)

2. V.P. Kuzmina, Efficiency of mechanical activation application during the production of dry construction mixtures, Dry Building Mixtures 5(37), 26-29 (2013)

3. N.I. Alfimova, V.V. Kalatozi, S.V. Karatsupa, Mechanical activation as a way to increase the efficiency of using raw materials of various genesis in construction materials science, Bulletin of BSTU named after V.G. Shukhov $\mathbf{6}$, 85-89 (2016)

4. I.N. Kravchenko, A.N. Biryukov, V.P. Trostin, A.O. Fedorov, Modernization of technological equipment for the enrichment of local building materials and disposal of destroyed structures, Construction and road vehicles 12, 44-50 (2019)

5. I.N. Kravchenko, N.I. Salyaev, D.A. Bumarskov, Improving the technological reliability of equipment for roadbuilding complexes, Mechanization of construction 12(858), 9-15 (2015)

6. I.N. Kravchenko, A.O. Fedorov, Yu.A. Biryukov, A.N. Biryukov, V.P. Trostin, The use of digital design technologies for the selection of rational technical solutions for mobile road construction complexes, Construction and road vehicles $1,35-44$ (2020)

7. V.P. Kuzmina, Vibratory centrifugal mills for mechanical activation of semi-finished products of dry construction mixtures, Construction Materials 4, 82-85 (2007)

8. V.N. Khetagurov, E.S. Kamenetsky, M.V. Gegelashvili, V.A. Pliev, The use of vertical centrifugal mills for grinding the lumpy part of flux materials in the preparation of sinter mixture. Proceedings of higher educational institutions, Series. Engineering Sciences 1, 74-77 (2018)

9. S.A. Chervyakov, L.N. Pashkin, A.I. Sapozhnikov, Trends in the improvement of cone crushers, Mining Machines and Automation 3, 29-31 (2004)

10. A.A. Vavilov, Simulation of production systems, Mechanical Engineering. Berlin. Technique, 416 (1983).

11. I.N. Kravchenko, V.P. Trostin, A.O. Fedorov, Yu.A. Biryukov, A.N. Biryukov, Physical-mechanical model of dry concentration of cements in impact aeroactivators, Construction and road vehicles 4, 45-51 (2020)

12. A.N. Biryukov, I.N. Kravchenko, Yu.A. Biryukov, V.P. Trostin, A.O. Fedorov, Aeroactivator. Patent 197675. IPC B28C 5/16. B28C 9/04. 2020107626. Declared 02.18.2020. Publ. 21.05.2020. Bul. 15. 